\title{
Current trends in drugs avoided in pregnancy
}

\author{
Bathala Anitha*, S. Malavika, B. Kumar, Yerikala Ramesh \\ Ratnam Institute of Pharmacy, Pidathapolur (V), Muthukur (M), S.P.S.R. Nellore 524346, Andhra Pradesh, India.
}

\begin{abstract}
During pregnancy several drugs are having contraindication, hence their use is less and dangerous to mother along with fetus .Drugs play an important role in improving the health and promoting well-being. However to produce desired effect, they have to be safe, efficacious and have to be used rationally. During pregnancy medication is less preferred but in some times cannot be escaped to treat the ailments in mother. Avoiding medications may be desirable, it is often not possible and may be dangerous because some women enter pregnancy medical conditions that require continuous and episodic treatment (e.g. asthma, hypertension, epilepsy). So here we discussed the medication that can be used safely during pregnancy along with unsafe and highly contraindicated for both mother and fetus. Certain drugs given during pregnancy may prove harmful to unborn child is one of the classical problem in the medical treatment. The main purpose of this review is to prepare a list of safe medications which can be taken during pregnancy with unsafe and highly contraindicated drugs. And also a quick reference for health care professionals.
\end{abstract}

Keywords: Current, Pregnancy, Drugs, Fetus

Article Info: Received 01 Oct, 2018; Review Completed 02 Nov 2018; Accepted 03 Nov 2018; Available online
15 Nov 2018
Cite this article as:
Anitha B, Malavika S, Kumar B, Ramesh Y, Current trends in drugs avoided in pregnancy, Journal of Drug Delivery and
Therapeutics. 2018; 8(6):342-350 D0I: http://dx.doi.org/10.22270/jddt.v8i6.2035

Bathala Anitha, Ratnam Institute of Pharmacy, Pidathapolur (V), Muthukur (M), S.P.S.R. Nellore 524346, Andhra Pradesh, India.

\section{INTRODUCTION}

Pregnancy occurs when sperm meets an egg. Pregnancy is a period in which a fetus is developed into a women's womb it contains one or more than one fetus ${ }^{1}$. It is known as gestation period in the blood (or) urine pregnancy is confirmed symptoms of early pregnancy are-nausea, vomiting, mood swing, missed period, tender breast [1]. It contains three trimesters, first trimester from 1-12 weeks, in this stage conceiving takes place and also miscarriage. Second trimester from 13-28 weeks, in this stage fetus movement can be felt. Third trimester from 29-40 weeks, in this sage parental care is very essential ${ }^{2}$. It has been reported that $8 \%$ of pregnant women need drug due to various chronic diseases and pregnancy related problems ${ }^{3}$. About $59 \%$ of pregnant women are needed a medication other than a vitamin or mineral supplements ${ }^{4}$. About $13 \%$ of pregnant women's are taking dietary herbal supplements. More than $90 \%$ of pregnant women take prescription or nonprescription drugs or use social drugs such as tobacco or alcohol or illicit drugs at sometime during pregnancy [4]. Pregnant women are normally excluded from clinical trials and results from animal studies need not apply to human population 5. Hence providing treatment to pregnant women is a problem. Fear of causing fetal harm and death by a given drug in pregnancy has raised many challenges to clinical research about the safety use of drugs in pregnancy ${ }^{5}$. Medication safety information in pregnancy is usually obtained from case reports, epidemiological studies and animal studies; all of which have some limitations that make determining risks of a drug use during pregnancy difficult ${ }^{6}$. The use medications in pregnancy should be evaluated by the benefits and risks to the mother and fetus 7 . Upon evaluation, some medications may be used sparingly during some trimesters contraindicated in others. All efforts should be made to optimize the risk-benefit ratio. It's very important for the pregnant mothers to follow up of do's and don'ts during the gestation period 7. The most important thing is the medication containing the mixture of chemicals, when interact together may cause the teratogenic effect to the baby ${ }^{8}$. So it is very important for the health care professionals and pregnant woman to know which drugs can be take and which should not take during this period [8].

\section{Principles of therapy in pregnancy: ${ }^{9}$}

Prescribe drugs only when clearly indicated, through weighing benefits to mother against the risks to the fetus, based on the stage of pregnancy and drug information the drug should be selected, give the drug wih low effective doses and for the shortest effective time, an older and safe drug is preferred over a newer drug during first trimester, 
provide counseling to pregnant women about the use of immunizations during pregnancy, should be avoided Live vaccines-possible harmful effects to fetus. (measles, mumps, polio, rubella). Toxoids (diphtheria, tetanus) and inactive virus vaccines ( influenza, rabies, hepatitis B ) are considered safe for use, Who are attacked by hepatitis B, rabies, tetanus, or varicella, hyper immune globulins (IGIVs) can be given to pregnant women, IV administration of hyper immune globulins reduce the risk of infection.[19]

\section{Principles of therapy in lactation: 10,11}

Give drugs only when needed, avoid taking the contraindicated drugs or stop breast feeding, drugs taken by the systemic route can reach the infant in breast milk, lowest effective doses and for the shortest effective time should be prescribed, Stopping the breast feeding during maternal drug therapy is not recommended unless necessary, Women with HIV infection should not breast feed. Transmitted to the nursing infant, in some cases, mother may discard milk while receiving therapeutic drugs, to maintain lactation.

\section{Physiological changes: 12}

Weight increases and changes body shape (due to increases in breast tissue, blood volume in the extra vascular and extra cellular fluid), the average weight gain in pregnancy is $12.5 \mathrm{Kg}$. During normal pregnancy $1 \mathrm{Kg}$ weight due to protein, ${ }^{13}$ The rate of albumin production increased but, plasma albumin levels are decreased( due to increased plasma volume), fibrinogen levels are increased and total body fat also increased. The ratio of LDL and HDL increases in pregnancy. ${ }^{14}$ Renal blood flow increased and glomerular filtration rate secondary to increased to cardiac output.

\section{Pharmacokinetic changes: ${ }^{15,16}$}

The drug effect can be changed by the changes pharmacokinetic in pregnancy, hydrophilic drugs are more diluted and distributed In non pregnant women than pregnant women, Increased dose may require, Hydrophobic are more soluble in pregnant women, The free drug have therapeutic or adverse effects on the mother and for placental transfer to the fetus, excretion of drugs increased by kidneys, mainly which are excreted primarily unchanged in the urine (digoxin, lithium), In the pregnancy, the increased size of uterus decreased renal blood flow in supine position, This results in decreased excretion and prolonged effects of renally excreted drugs.

\section{Drug effects on the fetus: 17, 18, 19, 20}

The rate transfer drug depends on the chemical properties of drug such as protein binding, $\mathrm{pH}$ difference, lipid solubility and molecular weight of drug, only free unbound drug crosses the placenta, during pregnancy maternal albumin level are decreases while fetal albumin increases. As a result concentration of free drug increases which cross the placenta to reach the fetus, Fetal $\mathrm{pH}$ is slightly more acidic than maternal $\mathrm{pH}$ and so weak bases are more likely crossed the placenta, Lipid soluble drugs can easily diffuse across the placenta, Drug with low molecular weight $<<500$ $\mathrm{g} / \mathrm{mol}$ )diffuse freely across the placenta, Drugs with low molecular weight ( $500-1000 \mathrm{~g} / \mathrm{mol}$ ) cross the placenta les easily, Drugs with high molecular weight $(>1000 \mathrm{~g} / \mathrm{mol})$ do no cross he placenta, Trans placental transfer drugs increase in the third trimester due to increased maternal and placental blood flow, decreased thickness and increased surface of placenta, During first trimester, drug teratogenicity most likely occurs, when fetal organs are formed, During the second and third trimesters, drug adverse effects are: growth retardation, respiratory problems, infection, or bleeding.

\section{Drug categories in pregnancy:}

Drugs are teratogenic only at specific times during embryogenesis. Teratogenicity is a condition when any drug of chemical substance which produce deviations or abnormalities in the development of embryo. Therefore to avoid such problems it is very important to know which drugs should be prescribed during pregnancy ${ }^{21}$. Food and drug administration (1979) of America enforce the rule for the categorization of the drug that is contraindicated during pregnancy so a classification has been carried out as following.

The FDA has categorized the potential teratogenic risk of $m$ edications by an A, B, C, D, X system 22,23 , and 24 .

Category A: Controlled studies in women have failed to demonstrate a risk to the fetus in the first trimester and there is no evidence of risk in later trimesters. The pos sibility of fetal harm appears remote. Medications in this $\mathrm{cl}$ ass are considered safe to use in pregnancy. Examples of $m$ edications in this class are vitamins and levothyroxine.

Category B: Either animal reproduction studies have not demonstrated a fetal risk but there are no controlled studies in pregnant women, or animal studies have demonstrated risk to the fetus that was not confirmed in controlled studies in pregnant women in the first trimester and there is no evidence of a risk in later trimesters.

Medications in this class are generally considered safe. Examples of medications in this class are acetaminophen and amoxicillin.

Category C: Studies in animals have revealed adverse effec ts on the fetus and there are no controlled studies in wome $\mathrm{n}$, or studies in women and animals are not available. Drugs from this class can be given to pregnant women if the bene fit to the mother outweighs the risk to the fetus. Examples of medications in this class are diltiazem and spironolacton e.

Category D: Evidence of human fetal risk has been docume nted, but the benefits to the mother may be acceptable des pite the risk to the fetus. Drugs in this class may be used in pregnancy if the benefits to themother outweigh the risk to the fetus (i.e. a life threatening situation or a serious diseas e for which safermedication cannot be used or are not effic acious). Examples of medications in this class are phenytoi $\mathrm{n}$ and valproic acid.

\section{Category $\mathrm{X}$ :}

Studies in animals or humans have demonstrated teratogen ic effects. The risk to the fetus clearly outweighs any potential benefit to the mother. Drugs in this category are contraindicated in pregnancy. Examples of medications in this class are thalidomide and warfarin.

Command problems in pregnancy: 25, 26 Anemia, Constipation, Gastro esophageal reflux, Gestational diabetes, Nausea and vomiting, Hypertension. 
Table 1: List of Antibiotics used in pregnancy: 27, 28, 29

\begin{tabular}{|c|c|c|c|c|}
\hline Generic (brand) & $\begin{array}{l}\text { Pregnancy } \\
\text { Category }\end{array}$ & $\begin{array}{l}\text { Crosses } \\
\text { placenta }\end{array}$ & $\begin{array}{l}\text { Reported adverse effects to mom or } \\
\text { baby use in pregnancy }\end{array}$ & Place in therapy \\
\hline Nitrofurantoin & $\mathrm{B}$ & Yes & Fetus: Hemolytic anemia & ---- \\
\hline $\begin{array}{l}\text { Sulfamethoxazole } \\
\text { / trimethoprim }\end{array}$ & $\mathrm{C}$ & $\begin{array}{l}\text { SMX: } \\
\text { Unknown } \\
\text { TMP: Yes }\end{array}$ & $\begin{array}{l}\text { Fetus: SMX: jaundice, hemolytic anemia, an } \\
\text { d possibly kernicterus TMP: neural tube de } \\
\text { fects (NTD), oral clefts, cardiac defects, } \\
\text { and urinary tract defects }\end{array}$ & $\begin{array}{l}\text { Not recommended in } \\
\text { pregnancy }\end{array}$ \\
\hline $\begin{array}{l}\text { Meronidazole } \\
\text { (Flagyl) Topical:- } \\
\text { (metro gel) }\end{array}$ & B & Yes & $\begin{array}{l}\text { Fetus: Low birth weight babies, } \\
\text { spontaneous abortions, and carcinogenic } \\
\text { possibilities. Not mutagenic or teratogenic }\end{array}$ & $\begin{array}{l}\text { Safe for use only in } \\
2^{\text {nd }} \text { and } 3^{\text {rd }} \text { trimester } \\
\text { Contraindicated in } 1^{\text {st }} \\
\text { trimester }\end{array}$ \\
\hline Tetracycline's & $\mathrm{D}$ & Yes & $\begin{array}{l}\text { Fetus: Hypo spadia ( } 1^{\text {st }} \text { trimester only), } \\
\text { inguinal hernia, limb hypoplasia, teeth } \\
\text { discoloration }\left(2^{\text {nd }}, 3^{\text {rd }}\right) \text { Cataracts, cleft } \\
\text { palates, spine bifida, polydactyl, Maternal: } \\
\text { liver toxicity, irreversible shock }\end{array}$ & $\begin{array}{l}\text { No recommended in } \\
\text { pregnancy }\end{array}$ \\
\hline Fluoroquinolones & $\mathrm{C}$ & Yes & $\begin{array}{l}\text { Erosion of weight bearing cartilage in rats } \\
\text { and dogs, but no human reports }\end{array}$ & $\begin{array}{l}\text { Not recommended in } \\
\text { pregnancy }\end{array}$ \\
\hline Macrolides & $\begin{array}{l}\text { Azithro, } \\
\text { Erythro: B } \\
\text { Claritro: C } \\
\end{array}$ & Yes & $\begin{array}{l}\text { Fetus: Cardiovascular abnormalities and } \\
\text { cleft palate with clarithromcin }\end{array}$ & \\
\hline Clindamycin & B & Yes & $\begin{array}{l}\text { Fetus: Increase in neonatal infection and } \\
\text { low birth weight seen with vaginal } \\
\text { preparation }\end{array}$ & $\begin{array}{l}\text { For BV as oral } \\
\text { alternative, but not } \\
\text { the topical Group B } \\
\text { strep. disease in } \\
\text { patients with } \\
\text { penicillin allergy }\end{array}$ \\
\hline Cephalosporin's & B & Yes & None reported & $\begin{array}{l}\text { Generally considered } \\
\text { safe in pregnancy } \\
\text { unless penicillin } \\
\text { allergic }\end{array}$ \\
\hline $\begin{array}{l}\text { Penicillin's +/-Beta- } \\
\text { lactamase inhibitor }\end{array}$ & B & Yes & None reported & $\begin{array}{l}\text { Safest class of abx in } \\
\text { pregnancy if not } \\
\text { allergic for syphilis } \\
\end{array}$ \\
\hline $\begin{array}{l}\text { Amino glycosides } \\
\text { (Amikacin, } \\
\text { Gentamycin and } \\
\text { Tobramycin) }\end{array}$ & $\mathrm{D}$ & Yes & $\begin{array}{l}\text { Fetus: ototoxicity/ deafness } \\
\text { Neuromuscular weakness, respiratory } \\
\text { depression with concomitant gentamicin } \\
\text { and Magnesium sulfate }\end{array}$ & $\begin{array}{l}\text { Do not use in } \\
\text { pregnancy not unless } \\
\text { the benefit out } \\
\text { weight the risk to the } \\
\text { fetus. }\end{array}$ \\
\hline
\end{tabular}

Carbapenems: 30 Category B/C/B in pregnancy, likely cross the placenta, Very little human data

Lactation: Excreted into breast milk in low amount, Unknown effects but likely low clinical significance

Linezolid: Pregnancy Category C, No human data available

Lactation: Unknown, myelo suppression in animals

Chloramphenicol: Risk during pregnancy It is an antibiotic which is useful in serious infections such as typhoid fever. Not have any adverse effect but can cause 'grey baby syndrome' and reversible bone marrow when it is given just before the delivery.

\section{Anti tubercular Agent: 31}

Streptomycin: Anti tubercular drug. It causes a minor effect to the fetus after crossing the placenta. It is mainly given to whom are resistant to rifampicin.

Miscellaneous: Tetanus injection: Injectable preparation is administered during second and third trimester of pregnancy to prevent tetanus ${ }^{31}$.

Probenecid: It is administered along with the penicillin and it is safe to be used during pregnancy.

Calcium and Vitamin-D: These can be safely given in the deficiency states and in accurate doses. 
Table 2: List of Anti epileptic drugs 32, 33, 34, 35

\begin{tabular}{|c|c|c|c|c|}
\hline Generic (brand) & $\begin{array}{l}\text { Pregnancy } \\
\text { Category }\end{array}$ & $\begin{array}{l}\text { Crosses } \\
\text { placenta }\end{array}$ & $\begin{array}{l}\text { Reported adverse effects to } \\
\text { mom or baby use in pregnancy }\end{array}$ & Place in therapy \\
\hline $\begin{array}{l}\text { Carbamazepine } \\
\text { (Tegretol) }\end{array}$ & $\mathrm{D}$ & $\begin{array}{l}\text { Yes: levels } 50-8 \\
0 \% \\
\text { of maternal, hig } \\
\text { hest } \\
\text { in fetal liver an } \\
\text { d kidneys }\end{array}$ & $\begin{array}{l}\text { Fetus: dysmorphic facial feature, } \\
\text { cranial defects, cardiac defects, s } \\
\text { pina bifuda, fingernail } \\
\text { hypoplasia, developmental delay, } \\
\text { mild mental retardation, neural t } \\
\text { ube defect }\end{array}$ & $\begin{array}{l}\text { Compatible - } \\
\text { Maternal Benefit >> Embryo/Fet } \\
\text { al Risk If drug is required durin } \\
\text { g pregnancy it should not be with } \\
\text { held because the benefits ofpreve } \\
\text { nting seizures outweigh potential } \\
\text { fetal hard. }\end{array}$ \\
\hline $\begin{array}{l}\text { Ethosuximide } \\
\text { (Zarontin) }\end{array}$ & $\mathrm{C}$ & Unknown & $\begin{array}{l}\text { Fetus: spontaneous hemorrhage, } \\
\text { patent ductus arteriosus, cleft lip } \\
\text { /palate, mongoloid facies, short n } \\
\text { eck, altered palmar crease and ac } \\
\text { cessory nipple, hydrocephalu }\end{array}$ & $\begin{array}{l}\text { Limited human data. Probably co } \\
\text { mpatible. Succinamide anticonv } \\
\text { ulsants: DOC for tx of } \\
\text { petit mal epilepsy in 1st } \\
\text { Trimester. }\end{array}$ \\
\hline $\begin{array}{l}\text { Felbamate } \\
\text { (Felbatol) }\end{array}$ & $\mathrm{C}$ & Unknown & $\begin{array}{l}\text { Fetus: mental retardation. } \\
\text { Maternal: aplastic } \\
\text { anemia, acute liver failure }\end{array}$ & $\begin{array}{l}\text { Limited Human Data - } \\
\text { Animal Data Suggest Moderate Ri } \\
\text { sk. Drug crosses placenta in anim } \\
\text { als, not yet described in humans. } \\
\text { But should occur because } \\
\text { of LMW }\end{array}$ \\
\hline $\begin{array}{l}\text { Phenytoin } \\
\text { (Dilatin) }\end{array}$ & $\begin{array}{l}\text { D } \\
\text { Dose } \\
\text { related } \\
\text { teratogenic } \\
\text { effect }\end{array}$ & Unknown & $\begin{array}{l}\text { Fetus: congenital abnormalities, } \\
\text { hemorrhage at birth, } \\
\text { neurodevelopment } \\
\text { abnormalities } \\
\text { Maternal: folic acid deficiency }\end{array}$ & $\begin{array}{l}\text { Compatible - } \\
\text { Maternal Benefit >> } \\
\text { Embryo/Fetal Risk } \\
\text { Significant Risks: major/minor c } \\
\text { ongenital abnormalities, } \\
\text { hemorrhage at birth, } \\
\text { neurodevelopment } \\
\text { Maintain lowest level required to } \\
\text { prevent seizures in order to less } \\
\text { en risk of fetal anomalies }\end{array}$ \\
\hline $\begin{array}{l}\text { Fosphenatyoin } \\
\text { (Cerebyx) }\end{array}$ & $\mathrm{D}$ & Unknown & $\begin{array}{l}\text { Fetus: congenital malformations, } \\
\text { orofacial clefts, cardiac defects, m } \\
\text { inor anomalies, mental } \\
\text { deficiency } \\
\text { Maternal: An increase in seizure f } \\
\text { requency may occur during preg } \\
\text { nancy because of altered } \\
\text { phenytoin pharmacokinetics }\end{array}$ & $\begin{array}{l}\text { Benefits from use in pregnant wo } \\
\text { men may be acceptable } \\
\text { despite the risk (e.g., if the drug i } \\
\text { s needed in a life-threatening situ } \\
\text { ation or for a serious } \\
\text { disease for which safer drugs } \\
\text { cannot be used or are ineffective) }\end{array}$ \\
\hline $\begin{array}{l}\text { Gabapentin } \\
\text { (Neurontin) }\end{array}$ & $\mathrm{C}$ & Unknown & $\begin{array}{l}\text { Limited human data does not } \\
\text { allow an assessment as to the } \\
\text { safety of gabapentin }\end{array}$ & $\begin{array}{l}\text { Limited Evidence: If required, be } \\
\text { nefits appear > fetal risk }\end{array}$ \\
\hline $\begin{array}{l}\text { Lomotrigine } \\
\text { (Lamictal) }\end{array}$ & $\mathrm{C}$ & Yes & $\begin{array}{l}\text { Fetus: frequency of major defects } \\
\text { among } 1^{\text {st }} \text { trimester } \\
\text { monotherapy exposure was } 2.9 \% \\
(12 \text { of } 414)\end{array}$ & $\begin{array}{l}\text { Human data Suggest Low Risk; } \\
\text { Adjust dose maintain clinical } \\
\text { respons }\end{array}$ \\
\hline $\begin{array}{l}\text { Levitiracetam(ke } \\
\text { ppra) }\end{array}$ & $\mathrm{C}$ & Unknown & $\begin{array}{l}\text { Risk to human fetus/embryo unk } \\
\text { nown }\end{array}$ & $\begin{array}{l}\text { Risk to human embryo/fetus is u } \\
\text { nknown }\end{array}$ \\
\hline $\begin{array}{l}\text { Oxcarbamazepin } \\
\text { e (Trileptal) }\end{array}$ & $\mathrm{C}$ & Yes & $\begin{array}{l}\text { Fetus: no major congenital malfo } \\
\text { rmations reported, mild facial } \\
\text { defects observed in one cas }\end{array}$ & $\begin{array}{l}\text { No epoxide metabolites: lower } \\
\text { risk of teratogenicity compared } \\
\text { to other agents, Supplement } \\
\text { with folic acid }\end{array}$ \\
\hline $\begin{array}{l}\text { Phenobarbital } \\
\text { (Luminal } \\
\text { sodium) }\end{array}$ & $\mathrm{D}$ & Yes & $\begin{array}{l}\text { Fetus: congenital defects, } \\
\text { hemorrhage at birth, addiction, } \\
\text { AE of neurobehavioral } \\
\text { development } \\
\text { Maternal: Benefit > Risk }\end{array}$ & $\begin{array}{l}\text { Benefits > Risk during at lowest } \\
\text { effective level }\end{array}$ \\
\hline $\begin{array}{l}\text { Pregablin } \\
\text { (Lyrica) }\end{array}$ & $\mathrm{C}$ & Unknown & $\begin{array}{l}\text { Fetus: congenital defects, } \\
\text { hemorrhage at birth, addiction, } \\
\text { AE of neurobehavioral } \\
\text { development } \\
\text { Maternal: Benefit > Risk }\end{array}$ & $\begin{array}{l}\text { Use only if maternal benefit }>\text { fetal } \\
\text { risk }\end{array}$ \\
\hline $\begin{array}{l}\text { Tiagabine } \\
\text { (Gabitril) }\end{array}$ & $\mathrm{C}$ & Unknown & $\begin{array}{l}\text { Fetus: one incidence with unspec } \\
\text { ified malformations, otherwise } \\
\text { unknown }\end{array}$ & $\begin{array}{l}\text { Safest course: Avoid in 1st } \\
\text { trimester; later } \\
\text { trimesters unknown }\end{array}$ \\
\hline $\begin{array}{l}\text { Primidone } \\
\text { (Mysoline) }\end{array}$ & $\mathrm{D}$ & Unknown & $\begin{array}{l}\text { Newborn: neurologic } \\
\text { manifestations }\end{array}$ & $\begin{array}{l}\text { If benefits > risks (e.g., drug } \\
\text { needed in life- }\end{array}$ \\
\hline
\end{tabular}




\begin{tabular}{|c|c|c|c|c|}
\hline & & & $\begin{array}{l}\text { (overactivity /tumor); mechanis } \\
\text { m for hemorrhagic effects is due } \\
\text { to suppression of VitK-dependen } \\
\text { t clotting factors, recommend } \\
\text { administration of VitK to infant i } \\
\text { mmediately after birth }\end{array}$ & $\begin{array}{l}\text { threatening situation or serious } \\
\text { disease with no safer drug) }\end{array}$ \\
\hline $\begin{array}{l}\text { Topiramate } \\
\text { (Topamax) }\end{array}$ & $\mathrm{C}$ & Yes & $\begin{array}{l}\text { Hypospadias in males (relationsh } \\
\text { ip not established); Data too limit } \\
\text { ed to assess embryo/fetus risk }\end{array}$ & Avoid if possible in 1st trimester \\
\hline $\begin{array}{l}\text { Valproic } \\
\text { acid(Depakene) }\end{array}$ & $\mathrm{D}$ & Yes & $\begin{array}{l}\text { Fetus: neural tube defects, minor } \\
\text { facial defects, defects of the head, } \\
\text { face, digits, urogenital tract, men } \\
\text { tal and physical growth. }\end{array}$ & $\begin{array}{l}\text { Benefits > Risks (e.g., drug neede } \\
\text { d in life- threatening situation } \\
\text { or serious disease with no safer } \\
\text { drug) }\end{array}$ \\
\hline $\begin{array}{l}\text { Zonisamide } \\
\text { (Zonegran) }\end{array}$ & $\mathrm{C}$ & Unknown & Congenital anomalies possible & Avoid if possible in 1st trimester \\
\hline Trimithadione & $\mathrm{D}$ & Unknown & $\begin{array}{l}\text { Fetus: mental retardation, cranio } \\
\text { facial defects, genitourinary } \\
\text { defects, malformed hands, } \\
\text { clubfoot }\end{array}$ & Contraindicated in 1st trimester \\
\hline $\begin{array}{l}\text { Clonazepam } \\
\text { (klonopin) }\end{array}$ & $\mathrm{D}$ & Unknown & $\begin{array}{l}\text { Human data suggest low risk; fet } \\
\text { al and neonatal } \\
\text { toxicity has been reported }\end{array}$ & $\begin{array}{l}\text { Safest course is to avoid during } \\
\text { the 1st trimester; however, } \\
\text { if indicated, it should not be } \\
\text { withheld because of pregnancy }\end{array}$ \\
\hline $\begin{array}{l}\text { Lorazepam } \\
\text { (Ativan) }\end{array}$ & $\mathrm{D}$ & Yes & $\begin{array}{l}\text { Fetus: high IV doses may cause "f } \\
\text { loppy infant" } \quad \text { syndrome, higher } \\
\text { incidence of respiratory distress }\end{array}$ & $\begin{array}{l}\text { Benefits > Risks (e.g., drug neede } \\
\text { d in life- threatening situation } \\
\text { or serious disease with no } \\
\text { safer drug) }\end{array}$ \\
\hline $\begin{array}{l}\text { Carbamazepine( } \\
\text { Tegretol) }\end{array}$ & $\mathrm{D}$ & Yes & $\begin{array}{l}\text { Fetus:minor craniofacial defects, } \\
\text { fingernail hypoplasia, } \\
\text { developmental delay, mild } \\
\text { mental retardation }\end{array}$ & If required, Benefits $>$ risks \\
\hline
\end{tabular}

Table 3: List of Diabetes mellitus 36

\begin{tabular}{|c|c|c|c|c|c|}
\hline $\begin{array}{l}\text { generic } \\
\text { (brand) }\end{array}$ & class & $\begin{array}{l}\text { pregnancy } \\
\text { category }\end{array}$ & $\begin{array}{l}\text { crosses } \\
\text { placenta }\end{array}$ & $\begin{array}{l}\text { reported adverse effects } \\
\text { to mom or baby from use } \\
\text { in pregnancy }\end{array}$ & place in therapy \\
\hline $\begin{array}{l}\text { Glyburide (Giabeta, } \\
\text { Micronase, } \\
\text { Glynase) }\end{array}$ & Sulfonylurea & $\mathrm{C}$ & Yes & $\begin{array}{l}\text { Possible ear defects in } 1^{\text {st }} \\
\text { trimester, fetal } \\
\text { hypoglycemia }\end{array}$ & $\begin{array}{l}\text { Insulin is recommended } \\
\text { first line by the ADA; } \\
\text { ACOG recommends } \\
\text { use of this agent in } \\
\text { D2 or GDM }\end{array}$ \\
\hline $\begin{array}{l}\text { Glipizide } \\
\text { (Glucorol) }\end{array}$ & Sulfonylurea & $\mathrm{C}$ & Yes & $\begin{array}{l}\text { Possible ear defects in } 1 \text { st t } \\
\text { rimester, no teratogenicity } \\
\text { in animal studies }\end{array}$ & $\begin{array}{l}\text { Not recommended; limit } \\
\text { ed human data }\end{array}$ \\
\hline $\begin{array}{l}\text { Glimipiride } \\
\text { (Amaryl) }\end{array}$ & Sulfonylurea & $\mathrm{C}$ & Unknown & $\begin{array}{l}\text { Skeletal malformation in hi } \\
\text { gh doses }\end{array}$ & $\begin{array}{l}\text { Not recommended; No } \\
\text { human data }\end{array}$ \\
\hline $\begin{array}{l}\text { Metformin } \\
\text { (Glucophage,Forta } \\
\text { met,Glumeza) }\end{array}$ & Biguanide & B & Unknown & $\begin{array}{l}\text { Neural tube defects in anim } \\
\text { als at high doses. } \\
\text { Few abnormalities in huma } \\
\text { ns at normal doses and } \\
\text { likely due to poor BG } \\
\text { control }\end{array}$ & $\begin{array}{l}\text { Insulin is recommended } \\
\text { first line by the ADA; } \\
\text { ACOG recommends } \\
\text { use of this agent in D2 or } \\
\text { GDM }\end{array}$ \\
\hline $\begin{array}{l}\text { Sitagliptin } \\
\text { (Januvia) }\end{array}$ & $\begin{array}{l}\text { Dipeptidyl } \\
\text { peptidase IV } \\
\text { inhibitor }\end{array}$ & B & Unknown & $\begin{array}{l}\text { No good studies in humans; } \\
\text { animal studies show no } \\
\text { defects/complication at } \\
\text { high dose }\end{array}$ & Possible; No human data \\
\hline $\begin{array}{l}\text { Pioglitazone } \\
\text { (Actos) }\end{array}$ & TZD & $\mathrm{C}$ & Unknown & $\begin{array}{l}\text { Developmental delay, decr } \\
\text { eased fetal weight in } \\
\text { animals }\end{array}$ & Not recommended \\
\hline $\begin{array}{l}\text { Rosiglitazone } \\
\text { (Avandia) }\end{array}$ & TZD & $\mathrm{C}$ & Yes & $\begin{array}{l}\text { Fetal death/retardation wa } \\
\text { s seen in animal studies }\end{array}$ & Not recommended \\
\hline $\begin{array}{l}\text { Exenatide } \\
\text { (Byetta) }\end{array}$ & $\begin{array}{l}\text { Incretin } \\
\text { mimetic }\end{array}$ & $\mathrm{C}$ & Unknown & $\begin{array}{l}\text { Decreased fetal growth, ske } \\
\text { letal malformations in ani } \\
\text { mal studies }\end{array}$ & Not recommended \\
\hline $\begin{array}{l}\text { Pramlintide } \\
\text { (Symlin) }\end{array}$ & $\begin{array}{l}\text { Amylino } \\
\text { mimetic }\end{array}$ & $\mathrm{C}$ & Unknown & $\begin{array}{l}\text { Animals: neural tube defect } \\
\text { s, cleft palate at high doses }\end{array}$ & Not recommended \\
\hline Regular insulin & Short acting & $\mathrm{B}$ & NO & None reported & Drug of choice \\
\hline
\end{tabular}




\begin{tabular}{|c|c|c|c|c|c|}
\hline $\begin{array}{l}\text { (HumulinR, Novolin } \\
\text { R) }\end{array}$ & insulin & & & & \\
\hline $\begin{array}{l}\text { Lispro insulin } \\
\text { (Humalog) }\end{array}$ & $\begin{array}{l}\text { Rapid acting } \\
\text { insulin }\end{array}$ & B & NO & $\begin{array}{l}\text { Case reports: sudden neon } \\
\text { atal death, growth } \\
\text { retardation; controlled stu } \\
\text { dies: as efficacious as } \\
\text { regular insulin }\end{array}$ & Recommended \\
\hline $\begin{array}{l}\text { Glulisine insulin } \\
\text { (Apidra) }\end{array}$ & $\begin{array}{l}\text { Rapid acting } \\
\text { insulin }\end{array}$ & $\mathrm{C}$ & Unknown & No available studies & $\begin{array}{l}\text { Not recommended unles } \\
\mathrm{s} \text { benefits }>\text { risks }\end{array}$ \\
\hline $\begin{array}{l}\text { NPH insulin } \\
\text { (Humulin N, } \\
\text { Novolin N) }\end{array}$ & $\begin{array}{l}\text { Intermediae } \\
\text { acting }\end{array}$ & B & NO & None reported & Recommended \\
\hline $\begin{array}{l}\text { Glargine insulin } \\
\text { (Lantus) }\end{array}$ & Long acting & $\mathrm{C}$ & Unknown & No available studies & $\begin{array}{l}\text { Not recommended } \\
\text { unless benefits }>\text { risks }\end{array}$ \\
\hline $\begin{array}{l}\text { Detemir insulin } \\
\text { (Levemir) }\end{array}$ & $\begin{array}{l}\text { Intermediae } \\
\text { long acing }\end{array}$ & $\mathrm{C}$ & Unknown & $\begin{array}{l}\text { Visceral abnormalities } \\
\text { were seen in animals }\end{array}$ & Not recommended \\
\hline
\end{tabular}

Table 4: List of Analgesics Drugs 37, 38, 39, 40

\begin{tabular}{|c|c|c|c|c|c|}
\hline $\begin{array}{l}\text { Generic } \\
\text { (brand) }\end{array}$ & Class & $\begin{array}{l}\text { Pregnancy } \\
\text { category }\end{array}$ & $\begin{array}{l}\text { Crosses } \\
\text { placenta }\end{array}$ & $\begin{array}{l}\text { Reported adverse effects to } \\
\text { mom or baby from use in } \\
\text { pregnancy }\end{array}$ & $\begin{array}{l}\text { Place in } \\
\text { Therapy }\end{array}$ \\
\hline $\begin{array}{l}\text { Aspirin } \\
\text { (Bufferin, } \\
\text { Ecotrin) }\end{array}$ & NSAID & $\mathrm{C}$ & Yes & $\begin{array}{l}\text { Fetal: increased perinatal } \\
\text { mortaility, teratogenic } \\
\text { effects, pulmonary HTN, } \\
\text { bleeding risk, premature ductus } \\
\text { arteriosis closure } \\
\text { Maternal: anemia, ante/post partu } \\
\text { m hemorrhage, prolonged labour }\end{array}$ & $\begin{array}{l}\text { Should not be used in } \\
\text { pregnancy, consider } \\
\text { acetaminophen }\end{array}$ \\
\hline $\begin{array}{l}\text { Ibuprofen } \\
\text { (Advil, Midol) }\end{array}$ & NSAID & $\begin{array}{l}\text { B D In 3rd } \\
\text { trimester }\end{array}$ & UNKNOWN & $\begin{array}{l}\text { Fetal: ductus arteriosis constrictio } \\
\text { n,Pulmonary HTN in 3rd trimester, } \\
\text { Maternal: prolonged labor, } \\
\text { spontaneous abortion }\end{array}$ & $\begin{array}{l}\text { Should be avoided when } \\
\text { possible and completely } \\
\text { avoided during the 3rd } \\
\text { trimester. Consider acet } \\
\text { aminophen }\end{array}$ \\
\hline $\begin{array}{l}\text { Naproxen } \\
\text { (Aleve, } \\
\text { Anaprox, } \\
\text { Midol,Naprosy } \\
\text { n,Pamprin) }\end{array}$ & NSAID & $\begin{array}{l}\text { B; D In 3rd } \\
\text { trimester }\end{array}$ & Yes & $\begin{array}{l}\text { Fetal: ductus arteriosis } \\
\text { constriction, intracranial } \\
\text { hemorrhage, primary } \\
\text { pulmonary HTN }\end{array}$ & $\begin{array}{l}\text { Should be avoided when } \\
\text { possible and completely } \\
\text { avoided during the 3rd } \\
\text { trimester. } \\
\text { Consider acetaminophen. }\end{array}$ \\
\hline $\begin{array}{l}\text { Acetaminophe } \\
\mathrm{n}\end{array}$ & $\begin{array}{l}\text { Analgesic } \\
\text { antipyreti } \\
\text { c }\end{array}$ & B & Yes & $\begin{array}{l}\text { Fetal: overdose can lead to liver } \\
\text { toxicity Maternal: } \\
\text { overdose can lead to liver toxicity }\end{array}$ & $\begin{array}{l}\text { Drug of choice for analge } \\
\text { sia and fever } \\
\text { during pregnancy }\end{array}$ \\
\hline $\begin{array}{l}\text { Butorphanol } \\
\text { (Stadol) }\end{array}$ & $\begin{array}{l}\text { Narcotic } \\
\text { analgesic }\end{array}$ & $\begin{array}{l}\mathrm{C} \text { D if } \\
\text { prolonged use }\end{array}$ & Yes & $\begin{array}{l}\text { Fetal: sinusoidal fetal heart rate } \\
\text { pattern, addiction, respiratory } \\
\text { depression } \\
\text { Maternal - addiction }\end{array}$ & $\begin{array}{l}\text { Used for analgesia during } \\
\text { labor }\end{array}$ \\
\hline $\begin{array}{l}\text { Morphine ( } \\
\text { Duramorph,Ka } \\
\text { dian. }\end{array}$ & $\begin{array}{l}\text { Narcotic } \\
\text { analgesic }\end{array}$ & $\begin{array}{l}\text { C; D if } \\
\text { prolonged use }\end{array}$ & Yes & $\begin{array}{l}\text { Fetal: addiction, possible relation } \\
\text { to inguinal hernia and } \\
\text { respiratory depression } \\
\text { Maternal: addiction }\end{array}$ & $\begin{array}{l}\text { Should only be used } \\
\text { when analgesia } \\
\text { or anesthetic is clearly in } \\
\text { dicated }\end{array}$ \\
\hline $\begin{array}{l}\text { Fentanyl } \\
\text { (Actiq, } \\
\text { Duragesic) }\end{array}$ & $\begin{array}{l}\text { Narcotic } \\
\text { analgesic }\end{array}$ & $\begin{array}{l}\text { C; D if } \\
\text { prolonged use }\end{array}$ & Yes & $\begin{array}{l}\text { Fetal: respiratory depression, } \\
\text { dependence and loss of fetal } \\
\text { heart rate variability without } \\
\text { hypoxia }\end{array}$ & $\begin{array}{l}\text { Only use when benefits > } \\
\text { risks }\end{array}$ \\
\hline $\begin{array}{l}\text { Hdromorphon } \\
\text { e (Dilaudid) }\end{array}$ & $\begin{array}{l}\text { Narcotic } \\
\text { analgesic }\end{array}$ & $\begin{array}{l}\text { C D if } \\
\text { prolonged use }\end{array}$ & Yes & Fetal: respiratory depression & $\begin{array}{l}\text { Only use when benefits > } \\
\text { risks } \\
\text { Manufacturer recommen } \\
\text { ded CI in pregnancy }\end{array}$ \\
\hline $\begin{array}{l}\text { Tramado } \\
\text { (ultram) }\end{array}$ & $\begin{array}{l}\text { Narcotic } \\
\text { analgesic }\end{array}$ & $\mathrm{C}$ & Yes & $\begin{array}{l}\text { Fetal: dose related fetal toxicity in } \\
\text { animals, respiratory depression } \\
\text { and addiction }\end{array}$ & $\begin{array}{l}\text { Should be avoided until } \\
\text { further evidence concern } \\
\text { ing the dose related } \\
\text { fetal toxicity is available }\end{array}$ \\
\hline $\begin{array}{l}\text { Ergotamine[Er } \\
\text { gomar] }\end{array}$ & $\begin{array}{l}\text { Sympatho } \\
\text { lytic }\end{array}$ & $\mathrm{X}$ & Yes & $\begin{array}{l}\text { Fetal: increase uterine tone leading } \\
\text { to fetal hypoxia, teratogenic and } \\
\text { fetal toxicity }\end{array}$ & Do not use in pregnancy \\
\hline
\end{tabular}


Table 5: Cough and cold medications in pregnancy and lactation 41, 42, 43

\begin{tabular}{|c|c|c|}
\hline Drug or Drug Class & Pregnancy & Lactation \\
\hline Decongestants & $\begin{array}{l}\text { Pseudoephedrine, in the lowest dose and } \\
\text { shortest duration possible is considered the } \\
\text { decongestant of choice. However it may be } \\
\text { prudent to avoid its use in the first trimester } \\
\text { of pregnancy. Oxymetazoline and } \\
\text { xylometazoline can be considered when used } \\
\text { at appropriate doses for short durations. }\end{array}$ & $\begin{array}{l}\text { Systemic decongestants are best } \\
\text { avoided if breast milk production is } \\
\text { poor or marginal. } \\
\text { Oxymetazoline and xylometazoline are } \\
\text { considered drugs of choice }\end{array}$ \\
\hline Antihistamines & $\begin{array}{l}\text { The first generation antihistamines, } \\
\text { especially chlorpheniramine, are preferred If } \\
\text { not tolerated or effective, second generation } \\
\text { agents such as loratadine can be } \\
\text { recommended. }\end{array}$ & $\begin{array}{l}\text { Due to possible adverse effects on the } \\
\text { infant from first generation } \\
\text { antihistamines, second generation } \\
\text { agents are preferred in lactating } \\
\text { mothers. }\end{array}$ \\
\hline Antitussives-Codeine & Codeine is best avoided during pregnancy. & $\begin{array}{l}\text { Avoid or limit codeine use in lactation } \\
\text { due to risk of infant toxicity. }\end{array}$ \\
\hline $\begin{array}{l}\text { Antitussives- } \\
\text { Dextromethorphan }\end{array}$ & $\begin{array}{l}\text { Dextromethorphan is the preferred } \\
\text { antitussive in both pregnancy and lactation. } \\
\text { Consider lack of evidence of efficacy. }\end{array}$ & $\begin{array}{l}\text { Dextromethorphan is the preferred } \\
\text { antitussive in both pregnancy and } \\
\text { lactation. Consider lack of evidence of } \\
\text { efficacy. }\end{array}$ \\
\hline Expectorant & $\begin{array}{l}\text { Guaifenesin is considered safe in pregnancy } \\
\text { and lactation. Consider lack of evidence of } \\
\text { efficacy. }\end{array}$ & $\begin{array}{l}\text { Guaifenesin is considered safe in } \\
\text { pregnancy and lactation. Consider lack } \\
\text { of evidence of efficacy. }\end{array}$ \\
\hline Analgesics-Aceaminophen & $\begin{array}{l}\text { Acetaminophen is considered the } \\
\text { analgesic/antipyretic of choice in both } \\
\text { pregnancy and breastfeeding. }\end{array}$ & $\begin{array}{l}\text { Acetaminophen is considered the } \\
\text { analgesic/antipyretic of choice in both } \\
\text { pregnancy and breastfeeding. }\end{array}$ \\
\hline $\begin{array}{l}\text { Analgesics-ASA and NSAIDS } \\
\text { (naproxen and ibuprofen) }\end{array}$ & $\begin{array}{l}\text { ASA and NSAIDs are considered compatible } \\
\text { with pregnancy in the first and second } \\
\text { trimester, but should be avoided in the third } \\
\text { trimester. } \\
\text { Low-dose ASA is considered to pose lower } \\
\text { risk during pregnancy and breastfeeding. }\end{array}$ & $\begin{array}{l}\text { Non-aspirin NSAIDs are generally } \\
\text { considered compatible during } \\
\text { breastfeeding and ibuprofen is the } \\
\text { NSAID of choice due to greatest safety } \\
\text { data. } \\
\text { Low-dose ASA is considered to pose } \\
\text { lower risk during pregnancy and } \\
\text { breastfeeding. }\end{array}$ \\
\hline Lozenges & $\begin{array}{l}\text { Medicated throat lozenges are considered } \\
\text { safe in pregnancy and breastfeeding. }\end{array}$ & $\begin{array}{l}\text { Medicated throat lozenges } \\
\text { considered safe in pregnancy and } \\
\text { breastfeeding. }\end{array}$ \\
\hline Herbs for Cough and Cold & $\begin{array}{l}\text { Echinacea and ginseng (all forms) should be } \\
\text { avoided during pregnancy and } \\
\text { breastfeeding. }\end{array}$ & $\begin{array}{l}\text { Echinacea and ginseng (all forms) } \\
\text { should be avoided during pregnancy and } \\
\text { breastfeeding. }\end{array}$ \\
\hline
\end{tabular}

Table 6: List of Drugs during Immunizations 44

\begin{tabular}{|c|c|c|c|c|c|}
\hline $\begin{array}{l}\text { generic } \\
\text { (brand) }\end{array}$ & Class & $\begin{array}{l}\text { pregnancy } \\
\text { category }\end{array}$ & $\begin{array}{l}\text { crosses } \\
\text { placenta }\end{array}$ & 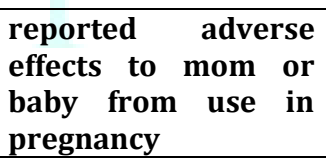 & $\begin{array}{l}\text { place in } \\
\text { therapy }\end{array}$ \\
\hline $\begin{array}{l}\text { Human } \\
\text { papillomavirus } \\
\text { (Gardasil) }\end{array}$ & $\begin{array}{l}\text { Inactivated } \\
\text { vaccine }\end{array}$ & B & Unknown & Currently under study & $\begin{array}{l}\text { Do not use during } \\
\text { pregnancy }\end{array}$ \\
\hline $\begin{array}{l}\text { Hepatitis } \\
\text { (Engerix-B, } \\
\text { Recombvivax HB) }\end{array}$ & $\begin{array}{l}\text { Inactivated } \\
\text { vaccine }\end{array}$ & $\mathrm{C}$ & Unknown & $\begin{array}{l}\text { No risk to the mom or } \\
\text { baby have been } \\
\text { reported }\end{array}$ & $\begin{array}{l}\text { The vaccine should } \\
\text { be given pre or post } \\
\text { exposure in women } \\
\text { at risk for infection. }\end{array}$ \\
\hline $\begin{array}{l}\text { Influenza } \\
\text { (injection)(Afluria, } \\
\text { Fluarix, } \\
\text { Flilaval,Fluvirin, } \\
\text { Fluzone) }\end{array}$ & $\begin{array}{l}\text { Inactivated } \\
\text { vaccine }\end{array}$ & $\mathrm{C}$ & Unknown & $\begin{array}{l}\text { Studies of immunizati } \\
\text { on of over } 2000 \\
\text { women showed } \\
\text { no fetal adverse } \\
\text { effects } \\
\text { associated with vaccin } \\
\text { ation }\end{array}$ & $\begin{array}{l}\text { ACOG recommends } \\
\text { the vaccine be given } \\
\text { to pregnant women } \\
\text { in the } 2 \text { nd and } 3^{\text {rd }} \\
\text { trimesters during flu } \\
\text { season. All at risk for } \\
\text { pulmonary } \\
\text { complications } \\
\text { should be vaccinated, } \\
\text { regardless of } \\
\text { trimester }\end{array}$ \\
\hline MMR (M-M-R II) & Live vaccine & $\mathrm{C}$ & Unknown & $\begin{array}{l}\text { Fetal infection with liv } \\
\text { e attenuated } \\
\text { virus may occur }\end{array}$ & $\begin{array}{l}\text { Do not use during pre } \\
\text { gnancy, Avoid } \\
\text { pregnancy for } 12\end{array}$ \\
\hline
\end{tabular}




\begin{tabular}{|l|l|l|l|l|l|}
\hline & & & & & \\
\hline $\begin{array}{l}\text { Pneumococcal } \\
\text { Vaccine } \\
\text { (Pneumovax) }\end{array}$ & $\begin{array}{l}\text { Inactivated } \\
\text { vaccine }\end{array}$ & $\mathrm{C}$ & $\begin{array}{l}\text { Maternal Ab } \\
\text { yes }\end{array}$ & $\begin{array}{l}\text { Risk to the fetus in the } \\
\text { 1st trimester is } \\
\text { unknown. }\end{array}$ & $\begin{array}{l}\text { Use if indicated in hig } \\
\text { h risk patients }\end{array}$ \\
\hline Td (Decavac) & Toxoid & $\mathrm{C}$ & Unknown & $\begin{array}{l}\text { No evidence of teratog } \\
\text { enicity }\end{array}$ & Use if indicated \\
\hline $\begin{array}{l}\text { TdP (Adacel, } \\
\text { Boostrix) }\end{array}$ & Toxoid & $\mathrm{C}$ & $\begin{array}{l}\text { Maternal Ab } \\
\text { yes }\end{array}$ & $\begin{array}{l}\text { Antibodies may also in } \\
\text { terfere with the infant' } \\
\text { simmune response to } \\
\text { infant doses of DTaP, s } \\
\text { o infant may not be pr } \\
\text { otected. }\end{array}$ & $\begin{array}{l}\text { Use if at high risk for } \\
\text { pertussiss }\end{array}$ \\
\hline $\begin{array}{l}\text { Varicella } \\
\text { Vaccine }\end{array}$ & Live vaccine & $\mathrm{C}$ & Unknown & $\begin{array}{l}\text { Fetal infection may } \\
\text { occur }\end{array}$ & $\begin{array}{l}\text { Do not use during } \\
\text { pregnancy }\end{array}$ \\
\hline
\end{tabular}

Social drugs: In addition to counsel the pregnant women regarding use of various prescribed and non- prescribed medications during pregnancy. They should be informed about risk of using following substances during pregnancy 45 .

Cigarette smoking: Maternal smoking is one of the few known preventable cause's prenatal morbidity and mortality ${ }^{46}$. The most consistent effect of smoking on the fetus during pregnancy is reduction in birth weight. Birth defects of heart, brain and face are also more common among babies of smokers. Risk of sudden infant death syndrome (SIDS), Mis-located placenta (placenta previa), premature detachment of placenta, premature rupture of the membranes, preterm labor, uterine infections, miscarriages, stillbirths, premature births are increased 47 . Changes in uterine and placental oxygenation may be the causes of infant death, pre-maturity or spontaneous abortions. Therefore all women's should be informed of the risk of smoking on the fetus and encouraged to quit smoking during pregnancy 48 .

Alcohol: Fetal alcohol syndrome is one of the most serious consequences of drinking during pregnancy ${ }^{49}$. Risk of miscarriage almost doubles for women who drink alcohol in any form during pregnancy and birth weight of babies is substantially below normal 50 . This syndrome includes inadequate growth before or after birth, facial defects, a small head, mental retardation and abnormal behavioral development.

Caffeine: Caffeine is found in various quantities in many beverages, analgesics, diet aids and stimulants; hence it is the most commonly ingested drug during pregnancy.

\section{REFERENCES}

[1] G Curtis, J Schuler (2000). Your Pregnancy Week by Week Fisher Books.

Availableat:https://books.google.com/books/about/Your_Pregnan cy_Week_by_Week.html?id=1pBXnVrW5nAC.

[2] RS Waddell, (2006). "FertilityPlus.org".Home Pregnancy Test hCG Levels and FAQ. [ONLINE] Available at: http://www.fertilityPlus.org/faq/hpt.html.

[3] The Sensible Guide to a healty preganancy. Available at: www.phac-aspc.gc.ca/hp-gs/pdf/hpguide- eng.pdf.

[4] Sharma R, Kapoor B, Verma U. Drug Utilization pattern during pregnancy in North India. J Med sci. 2006; 0:277-87.

[5] Andrade SE, Gurwitz JH, Davis RL, Chan KA, Finkelstein JA, F ortman K, et al. Prescription Drug Use Pregnancy. Am J ObstetGynaecol. 2004; 19.:398:407.
Evidence suggests that consuming caffeine during pregnancy possess little or no risk to the fetus. Caffeine contained in coffee, tea, and some sodas and some drugs is a stimulant that readily crosses the placenta to the fetus 51 . If taken in high dose it may stimulate the fetus increasing heart and breathing rate. Caffeine also may decrease blood flow across placenta and decreases the absorption of iron, increasing risk of anemia.

\section{CONCLUSION}

The safe and unsafe medications during pregnancy is a very important prospective of life as it carries the two lives conjoined for the certain period of time. During that time period both the mother and fetus should be safe, sound and grow healthily. This review summarizes the safe and unsafe list of drugs during pregnancy it is the responsibility of all clinicians including pharmacists to counsel patients with complete, accurate and current information on the risks and benefits of using medications during pregnancy. The first safe methods to refrain from such interaction during pregnancy are always consulting the medical practitioners and prescribe the drugs even of OTC category especially during the pregnancy as there are so many complications in it. It is the important that the benefits and risk of stopping treatment to be explained and informed properly. Drug may also be less effective during pregnancy because of pharmacokinetic changes such as increased metabolism. Doses of these drugs may need to be adjusting during pregnancy. Also when selecting drugs to be used in pregnancy effectively, drugs that have been in use for a long time are often preferable because fetal safety has been established even through newer alternatives may be available.

[6] Ward RW. Difficulties in the study of adverse fetal and neonatal effects of drug therapy during pregnancy.Semin Perinatol.2002; 25:191-5.

[7]TakingMedicineDuringPregnancy. Availableat:http://www.webmd.com/baby/guide/taking-medicineduring-pregnancy.

[8] Drugs contraindicated in pregnancy. Available at: http://www.empr.com/drugs contraindicated-inpregnancy/article/125914/9.

[9] Gerard G. Nahum, MD, CAPT Kathleen Uhl, USPHS, and CAPT Dianne L. Kennedy, USPHS.Antibiotic Use in Pregnancy and Lactation. American college of obstetricians and gynecologists. 107, 5, 2006.

[10] Trent Y.Antibiotic use in pregnancy. Infectious Diseases Registrar Cairns Base Hospital. 2011; 13(3). 
[11] Christina H. Stack R.Ph. The General Use of MedicationsinPregnancy ; Volume II, Number 8 | November/December 1999. 4-10.

[12] Moore PJ.Maternal Physiology during Pregnancy.In; De Cherney A, Pernoll ML, editors. Current obstetrics and gynecological diagnosis and treatment. $8^{\text {the }}$.New York: McGraw-Hill; 1994. pp.146-54

[13] Porter RS, editor. The Merk Manuals Online Medical Library. Whitehouse Staion: Merk Reaserch Lab; 2004

[14] Yankowitz J, NiebylJR, editors. Drug therapy for in pregnancy. $3^{\text {rd }}$ ed. philaadelphia: Lippincott William Wilkins; 2001.

[15] Hansen W, Yankowitz J, Nie by lJR. Pharmacological therapy for medical disorders during pregnancy. Clin Obstet Gynaecol. 2002 45:136-52.

[17] Refuerzo JS, Blackwell SC, Sokol RK, Use of over-the-counter medications and herbal remedies in pregnancy. Am J Perintatol 2005; $22: 321-4$

[18] Hale T, editor. Medications and mothers' milk. 12th ed. Amarillo TX: Pharmasoft Publishing; 2006.

[19] Briggs G, Freeman RK, Yaffe SJ, editors.Drugs in pregnancy and lactation. 7 th ed.: Lippincott

[20] Taking Medicine During Pregnancy. Available at: http://www.webmd.com/baby/guide/taking- medicine-duringpregnancy.

[21] L Bryant, T Fishman. J. Prim. Health Care, 2009; 1(2):150-151.

[22]Briggs GG, Freeman RK, Yaffe A. Drugs in Pregnancy and Lactati on: 7th Edition. Philadelphia: Lipppincott Williams \&

Wilkins; 2005.

[23]Micromedex Healthcare Series, (electronic version). Thomas Mi cromedex, Greenwood Village, Colorado, USA.

[24] Banhidy F, Lowry RB, Czeizel AE. Risk and benefits of drug use during pregnancy.Int J. Med Sci. 2005; 100-6.

[25] DeJong LT, Van Berg PB. A study of drug utilization during pregnancy in the light of known risks.Int J Risk Safety Med.1990; 1:91-105.

[26]Micromedex Healthcare Series, (electronic version). Thomas Mi cromedex, Greenwood Village, Colorado, USA.

[27] P Nand, KR Kher. A text book of hospital and clinical pharmacy, 13 th edition, Birla Publication Pvt limited, 2015, 292303 .

[28] JM Friedman, JE Polifka (2011). TERIS. Micromedex reproductive risk information system. Englewood, Colorado: Thomson MICROMEDEX.

[29] Trent Y.Antibiotic use in pregnancy.Infectious Diseases Registrar Cairns Base Hospital. 2011; 13(3).

[30] P Nand, KR Kher. A text book of hospital and clinical pharmacy, 13 th edition, Birla Publication Pvt limited, 2015, 292-303.

[31] Available at: http://www.wolterskluwercdi.com/.[Accessed 10, March 2016].

[32]Briggs GG, Freeman RK, Yaffe A. Drugs in Pregnancy and Lactati on: 7th Edition. Philadelphia: Lipppincott Williams \& Wilkins; 2005
[33]Micromedex Healthcare Series, (electronic version). Thomas Mi cromedex, Greenwood Village, Colorado, USA.

[34]Micromedex Healthcare Series, (electronic version). Thomas Mi cromedex, Greenwood Village, Colorado, USA.

[35]Briggs GG, Freeman RK, Yaffe A. Drugs in Pregnancy and Lactat on: 7th Edition. Philadelphia: Lipppincott Williams \& Wilkins; 2005

[36] Micromedex Healthcare Series, (electronic version). Thomas Mi cromedex, Greenwood Village, Colorado, USA.

[37] Christina H. Stack R.Ph. The General Use of Medicationsin Pregnancy ; Volume II, Number 8 | November/December 1999.

[38] Analgesics in pregnancy. Detail-Document; Canadian Pharmacist's Letter 2015; 22(3):310304

[39] Refuerzo JS, Blackwell SC, Sokol RK, et al. Use of over-thecounter medications and herbal remedies in pregnancy. Am J Perintatol 2005; 22: 321-324

[40] Natural Medicines Comprehensive Database.Therapeutic Research Faculty. 1995-2015. Available from:

http://naturaldatabase.therapeuticresearch.com/home.aspx?cs=\&s $=\mathrm{ND}$

[41] Grabenstein JD, Immunfacts 2008 Vaccines and Immunologic D rugs, Baltimore, MD: Williams \& Wilkins, 2007.

Centers for Disease Control.

[42] Pangle B. Drugs in pregnancy and lactation. In: Herfindsl ET, Gouley DR, ediors. Text book of Therapeutics, Drug and Diseases Management. $8^{\text {th }}$ ed. Philadelphia: Lippincort William Wilkins; 2006. pp.434-48

[43] Andrade SE, Gurwitz JH, Davis RL, Chan KA, Finkelstein JA, Fortman K, et al. Prescription ddrug use in pregnancy. Am J Obstet Gynaecol. 2004; 191:398-407

[44] Porter RS, editor. The Merk Manuals Online Medical Library. Whitehouse Staion: MerkReaserch Lab; 2004

[45] Pangle B. Drugs in pregnancy and lactation. In: Herfindsl ET, Gouley DR, ediors. Text book of Therapeutics, Drug and Diseases Management. $8^{\text {th }}$ ed. Philadelphia: Lippincort William Wilkins; 2006. 434-48

[46] Porter RS, editor. The Merk Manuals Online Medical Library. Whitehouse Staion: MerkReaserch Lab;2004

[47] Pangle B. Drugs in pregnancy and lactation. In: Herfindsl ET, Gouley DR, ediors. Text book of Therapeutics, Drug and Diseases Management. $8^{\text {th }}$ ed. Philadelphia: Lippincort William Wilkins; 2006. 434-48

[48] Pangle B. Drugs in pregnancy and lactation. In: Herfindsl ET, Gouley DR, ediors. Text book of Therapeutics, Drug and Diseases Management. $8^{\text {th }}$ ed. Philadelphia: Lippincort William Wilkins; 2006. 434-48

[49] Porter RS, editor. The Merk Manuals Online Medical Library. Whitehouse Staion: Merk Reaserch Lab; 2004

[50] Pangle B. Drugs in pregnancy and lactation. In: Herfindsl ET, Gouley DR, ediors. Text book of Therapeutics, Drug and Diseases Management. $8^{\text {th }}$ ed. Philadelphia: Lippincort William Wilkins; 2006. 434-48

[51] Porter RS, editor. The Merk Manuals Online Medical Library. Whitehouse Staion: Merk Reaserch Lab; 2004 